\title{
The formation and evolution of bulges as traced by globular cluster systems
}

\author{
Duncan A. Forbes ${ }^{1}$ and Lee Spitler ${ }^{1}$ \\ ${ }^{1}$ Centre for Astrophysics \& Supercomputing, Swinburne University, Hawthorn VIC 3122, \\ Australia \\ email: dforbes@swin.edu.au
}

\begin{abstract}
Globular Clusters provide a unique method for tracing the formation and evolution of their host galaxies. As single stellar populations they are far easier to interpret than the multi-population complexity of galaxy field stars. The scaling properties of globular clusters provide important constraints on the hierarchical assembly history of galaxies. Here we briefly review recent progress using the Hubble Space Telescope for imaging and the Keck plus Gemini telescopes for spectroscopy. We argue that the red, or metal-rich, subpopulation of GCs is associated with the bulge/spheroid component of galaxies. As one of the oldest stellar systems available for study, we discuss how globular clusters can be used to constrain the formation of galaxy bulges, in particular the role of mergers vs secular evolution. We conclude that metal-rich GCs, and hence bulges, formed very early in the Universe with more recent mergers having a small effect at most.
\end{abstract}

Keywords. globular clusters: general, galaxies: bulges, galaxies: evolution

\section{Introduction}

Most, perhaps all, large galaxies reveal two distinct subpopulations of globular clusters. These are defined in GC colour distributions to be blue and red subpopulations. As the vast bulk of GCs are old, the colours can be interpreted as metal-poor and metal-rich subpopulations. Both subpopulations 'know about' their host galaxy in the sense that the mean colour, or metallicity, correlates with galaxy luminosity (see Brodie \& Strader 2006 for a recent review). The slope and scatter of this relation provides constraints on the assembly history of galaxies.

The metal-rich GC subpopulation appears to be associated with the spheroid or bulge component in galaxies of all Hubble types. The slope of the GC metallicity vs galaxy luminosity relation for the metal-rich GCs is similar to that for the galaxy stellar metallicity vs luminosity relation. Additional support for the bulge/spheroid connection to metal-rich GCs is provided by detailed studies of individual galaxies. For example, in the elliptical galaxy NGC 4649 (Forbes et al. 2004) the g-i colour profile of the galaxy matches that of the red GCs at the same galactocentric radii. When we compare the surface brightness profile of the galaxy with the surface number density of red GCs we find a similar profile slope. Recent work on the elliptical NGC 3923 (Norris et al. 2007) reveals that the age and metallicity of the underlying galaxy light at the galactocentric radius of the red GCs is again similar.

In spiral galaxies, we also have good evidence that the red (metal-rich) GCs can be associated with the bulge component. Minniti (1995) and Cote (1999) showed that within the inner $5 \mathrm{kpc}$ of our Galaxy, the metal-rich GCs are spherically distributed with a 
similar mean metallicity, velocity dispersion and rotation to the bulge stars. Barmby et al. (2000) and Forbes et al. (2001) argued for a similar situation in M31 and M81. In the bulge-dominated Sa Sombrero galaxy, some $40 \%$ of the total GC population (2000) are metal-rich (800). This is some ten times the Galactic number even though the two galaxies have similar disk masses. The red GCs in Sombrero are spherically distributed and old (see Spitler et al. 2006). These facts argue strongly that the red GCs are not in general disk GCs but are bulge GCs.

\section{Bulge/Spheroid Formation and Evolution}

If red GCs are created in recent mergers (Ashman \& Zepf 1992), then we might expect the luminosity-weighted ages of the galaxy central stars to correlate with the mean colour of the red subpopulation (as the merger-formed red GCs will redden with the time since the merger event). Forbes et al. (2007) recently tested this prediction and found no correlation between mean red GC colour and galaxy 'age' for two different samples of elliptical galaxies. The data were consistent with a constant red colour. So although not a particularly sensitive test due to the age/metallicity degeneracy effects on colours, it did not support the idea of many red GCs forming in the last merger/interaction event (as traced by the central ages).

Spectroscopically derived ages are a much cleaner test. A number of ellipticals have been studied to date with sample sizes of a few tens of GCs. This includes NGC 1052 (Pierce et al. 2005) - a clear example of a young ( 2 Gyr), gaseous merger event and yet there are no signs from Keck spectra of 15 GCs for any of them to have formed along with the central galaxy's 2 Gyr old starburst.

A number of key galaxy properties show a distinct transition at a critical stellar mass of about $3 \times 10^{10} \mathrm{M}_{\odot}$. This mass has been interpreted as the transition from hot to cold accretion flows (e.g. Dekel \& Birnboim 2006). The number of GCs per unit galaxy luminosity, or stellar mass, also reveals this dichotomy exhibiting a U-shaped distribution (e.g. Forbes 2005). In Fig. 1 we show the ratio of the number of red GCs per unit bulge mass against bulge mass. Bulge masses come from 2MASS K-band luminosities and M/L ratios from stellar population models. Bulge-to-total ratios are assigned for each Hubble type (with a value of 1.0 assumed for ellipticals and the Sombrero galaxy). Different galaxy types are indicated on the plot. It can be seen that the bulges of spirals lie within the general trend defined by early-type galaxies. The effect of bulge growth by secular evolution would be to move the spirals down and to the right in this plot (as indicated by the arrow).

\section{Conclusions}

Globular cluster systems provide a useful probe of galaxy formation and evolutionary processes. We have argued that the metal-rich (red) GCs in a galaxy trace the spheroid component of early-type galaxies and the bulge component of spirals. Although recent accretions and gaseous mergers no doubt make some contribution, most metal-rich GCs formed early ( $\mathrm{z}>2$, age $>10$ Gyrs), ie spheriods/bulges formed early. The number of metal-rich GCs per unit bulge/spheroid stellar mass mirrors the dichotomy seen in other galaxy properties with a transition at a stellar mass of a few $10^{10} \mathrm{M}_{\odot}$. 


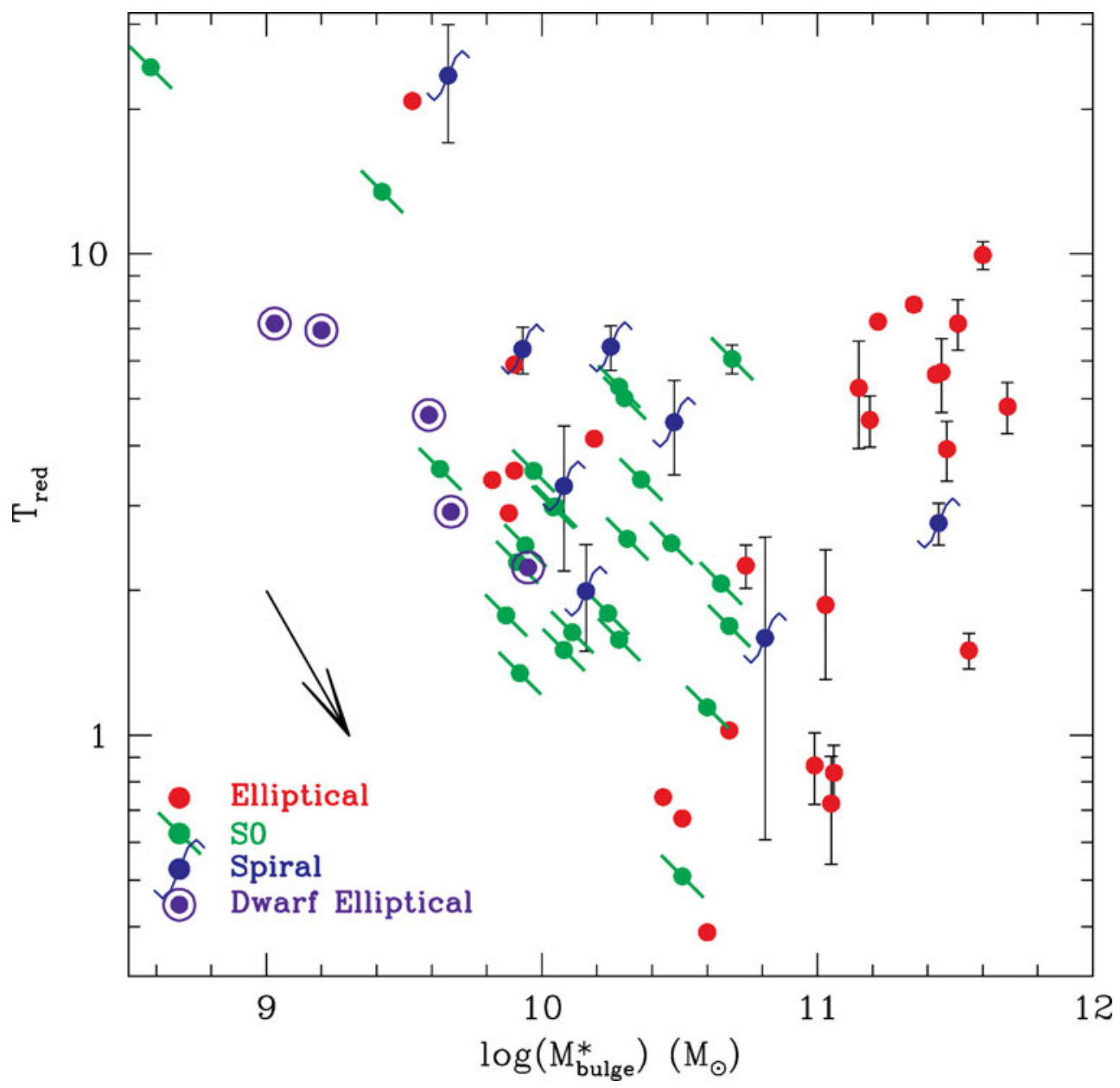

Figure 1. T parameter for red GCs against galaxy bulge mass. $\mathrm{T}_{\text {red }}$ is the number of red (metal-rich) GCs per unit galaxy bulge mass (where ellipticals are assumed to be all bulge). Bulgeto-total ratios are assigned for each Hubble type with bulge masses coming from 2MASS K-band luminosities and M/L ratios from stellar population models. The distribution is U-shaped with high $\mathrm{T}_{\text {red }}$ values at both high and low bulge masses. The critical transition mass occurs at a few times $10^{10} \mathrm{M}_{\odot}$. Bulge growth via secular evolution will tend to move the spiral galaxies down and to the right (as indicated by the arrow). Overall the bulges of spirals follow the same trend as the spheroid-dominated (elliptical) galaxies in terms of their $\mathrm{T}_{\text {red }}$ parameter.

\section{Acknowledgements}

DF would like to thank the ARC and the symposium organisers for financial support. We thank our colleagues in the SAGES project for their input and work on globular cluster systems over many years.

\section{References}

Ashman, K., Zepf, S. 1992, ApJ 348, 50

Barmby, P. 2000, $A J$ 119, 727

Brodie, J., Strader, J. 2006, ARAA 44, 193

Cote, P. 1999, AJ 118, 406

Dekel, A., Birnboim, Y. 2006, MNRAS 368, 2 
Forbes, D., et al. 2001, ApJL 556, 83

Forbes, D., et al. 2004, MNRAS 355, 608

Forbes, D. 2005, ApJL 635, 137

Forbes, D., et al. 2007, ApJ 659, 188

Minniti, D. 1995, AJ 109, 1663

Norris, M., et al. 2007, in prep.

Pierce M., et al. 2005, MNRAS 358, 419

Spitler., et al. 2006, AJ 132, 1593 